\title{
Investigating hemodynamic response variability at the group level using basis functions
}

\author{
Jason Steffener $^{\mathrm{a}, \mathrm{b}, *}$, Matthias Tabert ${ }^{\mathrm{c}, \mathrm{d}}$, Aaron Reuben ${ }^{\mathrm{a}, \mathrm{b}}$, Yaakov Stern ${ }^{\mathrm{a}, \mathrm{b}}$ \\ a Cognitive Neuroscience Division of the Taub Institute, Columbia University College of Physicians and Surgeons, New York, NY 10032, USA \\ b Department of Neurology, Columbia University College of Physicians and Surgeons, New York, NY 10032, USA \\ c Department of Psychiatry, Columbia University College of Physicians and Surgeons, New York, NY 10032, USA \\ d Department of Geriatric Psychiatry, New York State Psychiatric Institute, New York, NY 10032, USA
}

\section{A R T I C L E I N F O}

\section{Article history:}

Received 11 September 2009

Revised 5 November 2009

Accepted 6 November 2009

Available online 12 November 2009

\section{Keywords:}

fMRI

Hemodynamic response

Delay

Brain imaging

Basis sets

Group analysis

\begin{abstract}
A B S T R A C T
Introduced is a general framework for performing group-level analyses of fMRI data using any basis set of two functions (i.e., the canonical hemodynamic response function and its first derivative) to model the hemodynamic response to neural activity. The approach allows for flexible implementation of physiologically based restrictions on the results. Information from both basis functions is used at the group level and the limitations avoid physiologically ambiguous or implausible results. This allows for investigation of specific BOLD activity such as hemodynamic responses peaking within a specified temporal range (i.e., 4-5 s). The general nature of the presented approach allows for applications using basis sets specifically designed to investigate various physiologic phenomena, i.e., age-related variability in poststimulus undershoot, hemodynamic responses measured with cerebral blood flow imaging, or subject-specific basis sets. An example using data from a group of healthy young participants demonstrates the methods and the specific steps to study poststimulus variability are discussed. The approach is completely implemented within the general linear model and requires minimal programmatic calculations.
\end{abstract}

(c) 2009 Elsevier Inc. All rights reserved.

\section{Introduction}

Previous work has demonstrated that the hemodynamic response (HDR) to neural activity measured with blood oxygen level dependent (BOLD) fMRI varies across the brain and across individuals (Aguirre et al., 1998; Handwerker et al., 2004). This poses a problem for accurate quantification of an individual's BOLD activation when a canonical hemodynamic response function (HRF), i.e., a generalized model of the HDR is used. A simple solution to this problem has been to estimate an individual's HDR using simple visual or motor tasks (D'Esposito et al., 1999; Zarahn et al., 1997), resulting in an estimate of an individual's specific HRF model for use in their statistical analyses. Although this approach is an advance over the canonical HRF model, it has its own drawbacks: it requires additional scans, which cannot be acquired retrospectively, and fails to capture any intraindividual variance that may exist between the region used to derive the HRF and regions of interest for the main experiment. Therefore, this method still leaves potentially unaccounted for variance in the estimate of the BOLD HDR across regions of the brain.

One approach to capturing intraindividual variance in the BOLD HDR is with basis sets. Instead of a single function to model the HDR, a basis set uses multiple (typically two or three) related functions.

\footnotetext{
* Corresponding author. 630 West 168th St, P\&S 16 New York, NY 10032, USA. Fax: +1212342 1838 .

E-mail address: js2746@columbia.edu (J. Steffener).
}

Through weighted combinations of the functions, many HDR shapes may be modeled, allowing for investigations of hemodynamic variability in a variety of patient populations with various sensory stimuli (Ford et al., 2005; Handwerker et al., 2004; Salek-Haddadi et al., 2006; Stevens et al., 2005; Wierenga et al., 2008). The most prominent basis set currently used is derived from a series expansion of the standard 'double-gamma' HRF model (Friston et al., 1998; Glover, 1999). Other similar basis sets have been developed using a principal components analysis (PCA) of large sets of physiologically plausible BOLD HRFs (Friman et al., 2003; Liao et al., 2002; Woolrich et al., 2004). Of particular note is the work by Liao et al. (2002) who designed a basis set to be sensitive to large delays in the BOLD response to stimulation. This approach demonstrates the feasibility that basis sets may be developed to address specific physiological or clinical questions.

Unfortunately, one drawback of using any basis set is that many model fits result in physiologically ambiguous or implausible results (Calhoun et al., 2004; Woolrich et al., 2004). Restrictions on the potential model fits are therefore required to limit a basis set to plausible shapes. One approach to do so is to only investigate estimated HDRs that have a time to peak within a specific time window (Calhoun et al., 2004; Henson et al., 2002). While this approach may improve analyses at the individual level, translating these results to higher-level statistical analyses presents it own difficulties (Calhoun et al., 2004; Friman et al., 2003; Kiehl and Liddle, 2001; Worsley and Taylor, 2006). The main such difficulty regards the 
manner of excluding or including the variance accounted for by the multiple basis functions. One approach to deal with this variance is to fit a basis set and then only perform higher level analyses on the primary basis function, thus excluding the variance attributed to the secondary basis function(s) (Kiehl and Liddle, 2001). An alternative approach is to include the variance accounted for by all functions in the set at higher level analyses by using the magnitude calculated across the basis function estimates for higher level analyses (Calhoun et al., 2004).

The specific implementation of Calhoun et al. (2004) addresses some of the concerns of using a basis set for group-level analyses. This approach, however, is not general enough for use with other basis sets and requires specific normalization of the regressors in the model. The result is that the specific ratio described in their work is only meaningful with the basis set they used and would have a different meaning if directly applied to other basis sets, i.e., FLOBS as implemented with the FSL package. In addition, the canonical HRF, and its first derivative basis set, is specifically sensitive to temporal shifts, making it unsuitable for study of other physiological variations such as poststimulus undershoot variations. For instance, systematic differences in the size of the poststimulus undershoot between young and elder healthy participants were found in one study (Wierenga et al., 2008), while another study found no significant differences in the BOLD HDR shape between age groups (D'Esposito et al., 2003). In addition, growing application of BOLD fMRI to study disease states (Matthews et al., 2006) may introduce important variations in BOLD HDR shapes which need to be addressed. Specifically, studies of the hemodynamic responses to epileptiform discharges have shown large variability in the BOLD response as a function of proximity to the discharge site (Lemieux et al., 2008; Salek-Haddadi et al., 2006).

Importantly, the current advance of cerebral blood flow imaging ( $\mathrm{CBF}$ fMRI) also requires that many of the original questions regarding the shape and variability of hemodynamic responses be revisited with this hemodynamic marker. For instance, in a recent study using BOLD and CBF fMRI, age-related differences were found, within subject, in the poststimulus undershoot using CBF but not in BOLD (Ances et al., 2009). This suggests that the underlying hemodynamic response of CBF and BOLD fMRI differ and the same HRF model may not be optimal. Therefore, with CBF fMRI, new hemodynamic response models, and therefore basis sets, will need to be developed to be specifically sensitive to this new imaging modality (Woolrich et al., 2006; Yang et al., 2000). This is a clear instance in which there is a need for a general approach to group-level analyses with basis functions.

This technical note presents an approach using the general linear modeling framework (i.e., using the SPM or FSL packages) to address the two main limitations of using a basis set: the need for model fitting restrictions and the need for a means of translating individuallevel improvements in model fitting to higher-level analyses. The approach is straightforward and applicable to any basis set, allowing for flexible designations of physiological limitations, thereby correcting many of the difficulties presented when using basis sets. The specific normalizations of the design matrix required for this approach are calculated so that they may be performed after model estimation; therefore, no modifications are required to the design matrix as created by SPM or FSL. Furthermore, with such a general framework, it is possible to investigate more subtle questions regarding hemodynamic responses to neural activity. One potential application of this method will be to investigate the physiological origins of variations in the poststimulus undershoot measured with BOLD and CBF fMRI. An approach to addressing such a question is used as a specific example and laid out in detail later in the paper. Other applications will include the study of the spatial dynamics of BOLD responses to epileptic seizures (Lemieux et al., 2008) or investigation of the spatial variability of the temporal delay of BOLD responses. Data from a group of young participants engaged in a simple visual experiment are used to demonstrate the methods and software to implement the key calculations (for SPM or FSL) are posted at: http:/ / cumc.columbia.edu/dept/sergievsky/cnd/steffener.html.

\section{Methods}

\section{Participants}

Ten young healthy volunteers $(50 \%$ females; mean age $=$ 23.9 years \pm 5.4 ; education $=15.4 \pm 2.4$ years) participated in the fMRI study. The study was approved by the New York State Psychiatric Institute IRB and all subjects provided informed consent.

\section{fMRI parameters}

Scanning used a Philips Medical Systems Intera $1.5 \mathrm{~T}$ machine with echo planar imaging (EPI) capabilities (TR/TE $=3000 / 50 \mathrm{~ms}$, flip $=90^{\circ}$, slice thickness $=5 \mathrm{~mm}$ (no gap), 32 slices, orientation angle of $30^{\circ}$ to the AC-PC line, FOV $=20 \times 20 \mathrm{~cm}$, and a $64 \times 64$ matrix). High-resolution T1-SPGR images were acquired to aid in coregistration and anatomical localization (TR/TE $=25 / 3 \mathrm{~ms}$, flip $=45^{\circ}$, slice thickness $=2 \mathrm{~mm}$ (no gap), FOV $=23 \times 23 \mathrm{~cm}$, and a $256 \times 256$ matrix).

\section{Task description}

The visual task used a $2-\mathrm{Hz}$ reversing checkerboard for $12 \mathrm{~s}$ alternated with $30 \mathrm{~s}$ of fixation on a central cross-hair for five cycles projected to the center of the subject's field of view via a rear projection screen. A laptop computer (Dell 5150) using a customdeveloped program (LabView 7.1, National Instruments Corp.) presented the stimulus. An extra $6 \mathrm{~s}$ (2 scans) of data were acquired and discarded at the beginning of each functional run to account for MR saturation effects. The final result was 80 EPI scans comprising a single functional run.

\section{Image preprocessing}

All image preprocessing and statistical analyses were implemented using SPM5 (Wellcome Department of Cognitive Neurology). All EPI images were corrected for motion by realigning to the first volume of the series. The T1-weighted (structural) image was coregistered to the first EPI volume using mutual information. This coregistered highresolution image was then used to determine the linear and nonlinear parameters for transformation into a standard space defined by the Montreal Neurologic Institute (MNI) template brain supplied with SPM5. This transformation was then applied to the EPI data which were resliced using bilinear interpolation to $2 \times 2 \times 2 \mathrm{~mm}$ final voxel dimensions and spatially smoothed with an $8 \times 8 \times 8 \mathrm{~mm}$ FWHM (fullwidth at half-maximum) Gaussian kernel.

\section{Subject-level statistical modeling}

The functional data were modeled using a box-car representation of the stimulus vector convolved with the basis set consisting of the canonical double-gamma model of the HRF and its first derivative using SPM5's default parameters (Fig. 1A). The regression model using the basis set was therefore

$$
\begin{aligned}
& x_{1}=s^{* H R F} \\
& x_{2}=s^{*}\left(\frac{\partial H R F}{\partial t}\right) \\
& y_{t}=\beta_{0}+\beta_{1} \cdot x_{1}+\beta_{2} \cdot x_{2}+\varepsilon_{t}
\end{aligned}
$$



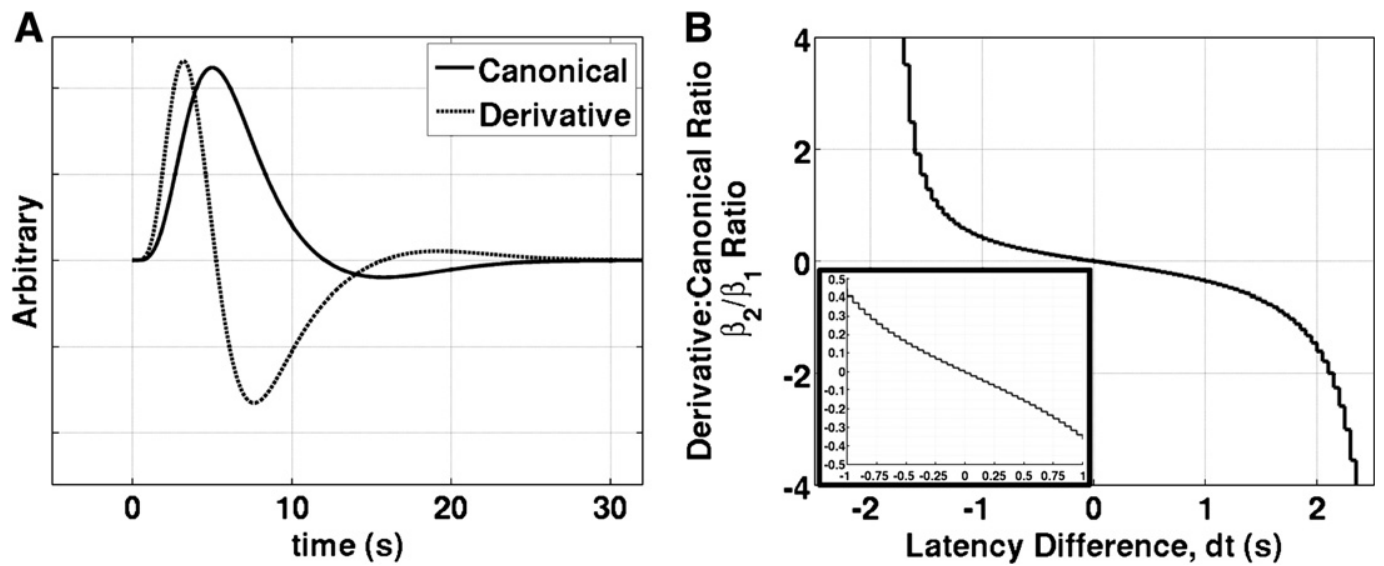

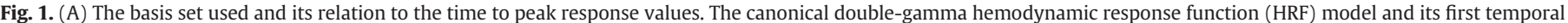

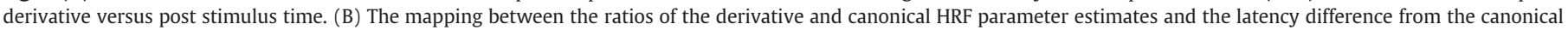
HRF's time to peak at $5 \mathrm{~s}$.

where $\mathbf{s}$ is the stimulus vector, * represents the convolution operation, $\mathrm{HRF}$ is the double-gamma model (see Appendix 1) of the hemodynamic response, $\frac{\partial \mathrm{HRF}}{\partial t}$ is its temporal derivative and $\varepsilon_{t}$ represents the residual noise assumed to be from an $\mathrm{AR}(1)$ process and accounted for in the GLM using SPM5's prewhitening procedure.

Once the model was fit to the data, the beta images (images of regression weights) for the basis set were combined by calculating the magnitude across both weights for each voxel (Calhoun et al., 2004):

$H=\sqrt{\left(\hat{\beta}_{1}^{2}+\hat{\beta}_{2}^{2}\right)}$

A similar value has previously been defined as the "derivative boost" (Lindquist et al., 2009). To apply this equation, however, the regressors need to be orthogonal and normalized to have sum of square values of 1 (Calhoun et al., 2004). If the regressors are not normalized by their sum of squares, this step can be performed via appropriately transformed contrast values after estimating the model. Appendix 1 has a full description of this procedure.

\section{Hemodynamic response extraction}

Estimates of the HDR were extracted to demonstrate the variability in the BOLD response across participants and brain regions. The parameter estimate maps for the regressor's corresponding to the canonical HRF and the temporal derivative terms were multiplied by high-resolution versions of the two basis functions:

$H \hat{D} R=H R F \cdot \hat{\beta}_{1}+\frac{\partial H R F}{\partial t} \cdot \hat{\beta}_{2}$

The result is that each voxel has a new time series which is the estimated HDR using the basis set. This allowed investigation of participant-specific and regionally specific variation in the estimated HDR. Mean estimated HDRs were then calculated for each participant and each hemisphere for Brodmann areas 17 and 18 of the visual cortex as defined by the Brodmann area map in standardized space provided with the software MriCro (Rorden and Brett, 2000; TzourioMazoyer et al., 2002).

\section{Basis set restrictions}

The steps for this procedure are laid out in the flowchart shown in Fig. 2. This flowchart will serve as a road map for the rest of the methods. The basis set chosen for this work, the canonical HRF, and its first derivative (step 1 in flowchart) are most sensitive to latency shifts relative to the canonical function (step 2) (Henson et al., 2002).
Restrictions on the responses were made with two aims in mind. First, responses had to be physiologically meaningful (e.g., having one peak) (Calhoun et al., 2004) and to be roughly within the linear range of the time to peak mapping (Fig. 1B) (Henson et al., 2002). Fig. 3 demonstrates examples of a full range of resultant HDR estimates from various ratios of the two basis functions. The two exemplars in boxes demonstrate ratios which result in responses which are difficult to interpret as either "activated" or "deactivated" (Calhoun et al., 2004).

Fig. $1 \mathrm{~B}$ maps the ratios of the derivative-canonical parameter estimates $\left(\beta_{2} / \beta_{1}\right)$ relative to the peak time of the primary basis function (latencies), which peaks at $5 \mathrm{~s}$ (step 3) (Henson et al., 2002). From this mapping, the relationship between the ratio of the basis functions and the latency time is approximately linear in the range of $\pm 1 \mathrm{~s}$. Using this relationship, only hemodynamic responses that occur within the range of peak times of 4 to $6 \mathrm{~s}$ are investigated.

Three time-to-peak ranges were therefore established for investigation: the full range of 4 to $6 \mathrm{~s}$, and the two halves: 4 to $5 \mathrm{~s}$ and 5 to $6 \mathrm{~s}$. This corresponds to identifying responses that occur within the ranges of the following: 1 ) later than $4 \mathrm{~s}, 2$ ) earlier than $6 \mathrm{~s}, 3$ ) earlier than $5 \mathrm{~s}$, and 4) later than $5 \mathrm{~s}$ (Table 1). The ratios for determining the time to peak limits were found from the mapping in Fig. 1B (step 4). The ratio values were then adjusted so that the norm of the two basis function weights equaled 1 (step 5). Contrast weights were then orthogonal vectors to these normalized weights depending on the range of interest (either early or later), see Appendix 2 for a detailed description of the calculation (step 6). As an example, a positive weight for the second basis function indicates a response occurring earlier than the primary basis function, see Fig. 3. Therefore, to investigate any response that occurs "early," a contrast with only a weight of 1 for the derivative term is appropriate. The result is all locations whose estimated response time to peak occurs earlier than the primary function's time to peak, in this case $5 \mathrm{~s}$. Flipping the sign on the contrast weights therefore results in all responses with peaks occurring later than $5 \mathrm{~s}$. Table 1 lists the time-to-peak ranges of interest, the ratios as determined from Fig. 1B, the normalized weights, and the required contrasts required for these ranges.

Subject-level analyses involved estimating the model, calculating the four contrasts listed in Table 1 (steps 7 and 8) and calculating the magnitude image using Eq. (4) (step 9). These five images were then used at the higher-level group analysis.

\section{Group-level analysis}

To restrict the group-level inference to be within the predefined limits, group-level analyses were first conducted on each contrast 

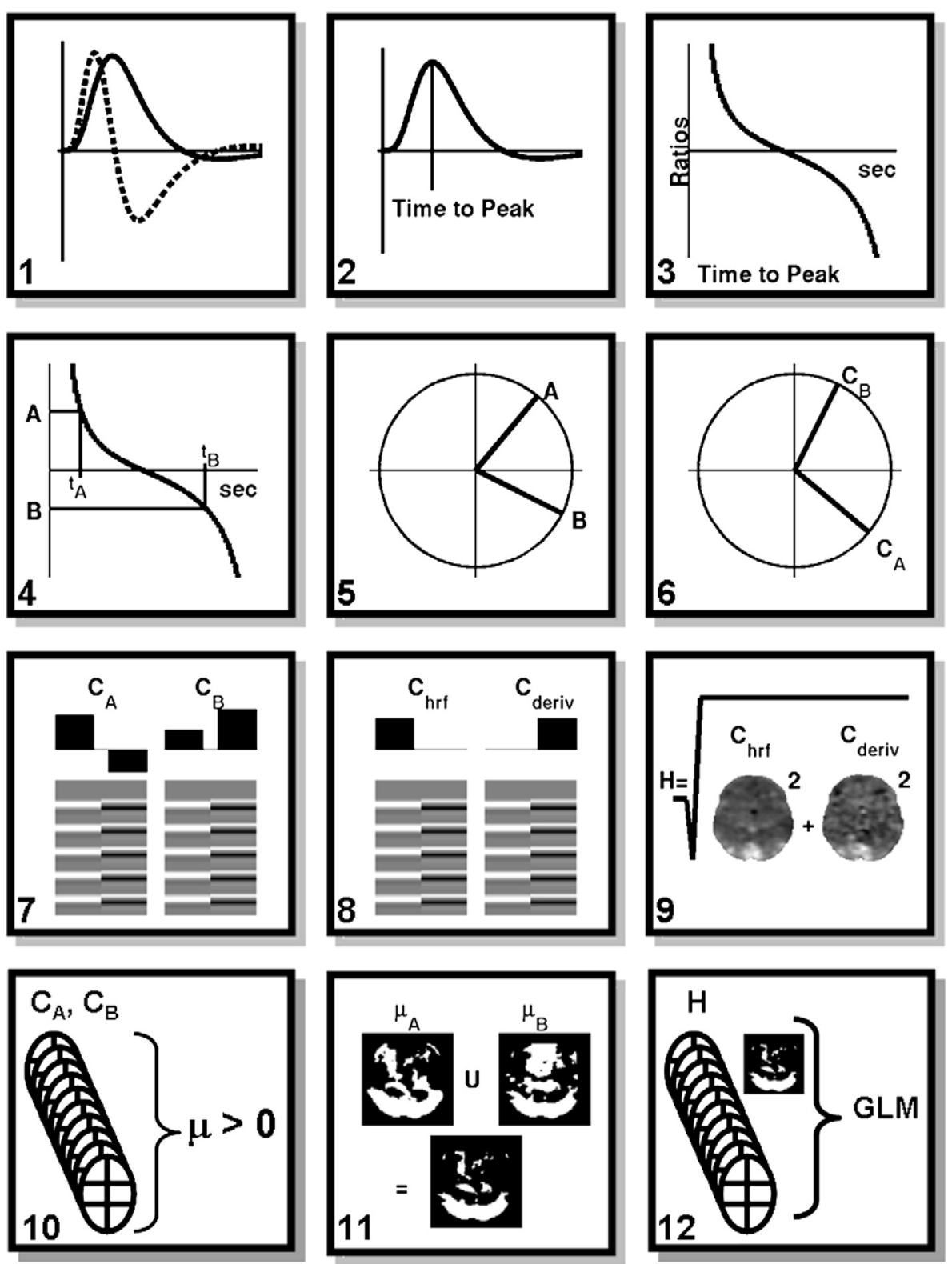

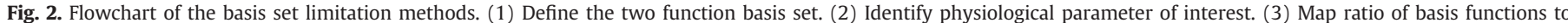

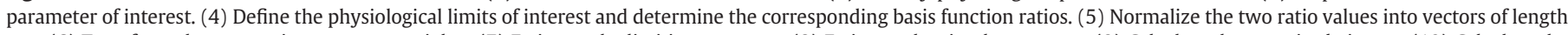

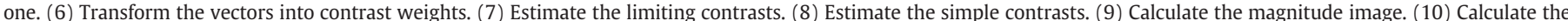

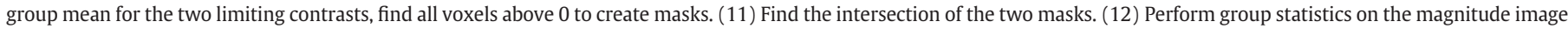
within voxels of the intersection mask.

described in Table 1 . This involved four one-sided, one-sample $t$-test group analyses, one for each contrast. Second, three masks were created, one for each range of interest (step 10). For the full time-topeak range of 4 to $6 \mathrm{~s}$, the resultant beta images (mean images for the group) for the 'later than $4 \mathrm{~s}$ ' contrast and the 'earlier than $6 \mathrm{~s}$ ' contrast images were masked for their intersection (step 11). Therefore, any voxels that had values greater than 0 in both images were included in the mask. Masks were created in the same manner for the ranges of 'later than $4 \mathrm{~s}$ ' and 'earlier than $5 \mathrm{~s}$ ' and for the range of 'later than $5 \mathrm{~s}$ ' and 'earlier than 6 s.'

Finally, one-sample t-test group analyses were performed on the magnitude images. This analysis was repeated three times using each of the three mask images as "exclusive" masks (step 12). Therefore, only those voxels which had group mean time to peak values in the specified ranges were investigated. Exclusive masking at the group-level ensures that each participant is contributing the same voxels to the group analysis. Statistical significance was defined with an FDR-corrected height threshold of $p<0.05$ and a cluster extent of 20 voxels.

\section{Results}

The HDR estimates for the two visual cortex regions of interest demonstrate the variability that the derivative term captures (Fig. 4). For most participants, the mean time to peak was earlier than the primary basis function's time to peak (Table 2). Therefore, the additional regressor in the model mainly accounted for early occurring HDRs. The variability in the time to peak estimates was not significantly correlated with age, education, nor did it differ by gender in any of the regions.

The group's mean results demonstrated extensive and highly significant task-related signal change throughout the visual cortex, see Fig. 5. A large proportion of the significant regions had responses that peaked between 4 and $5 \mathrm{~s}$ (early latencies). Early responses were 


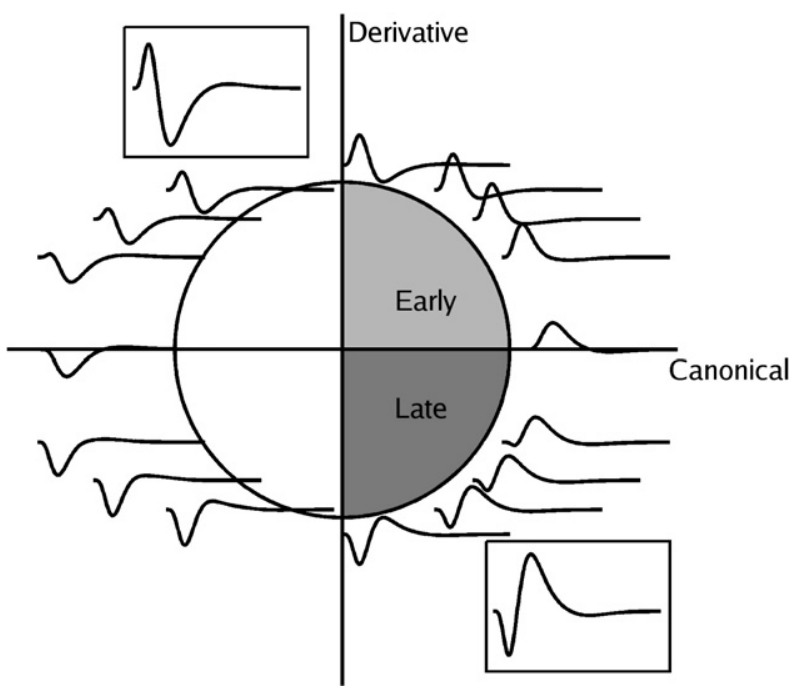

Fig. 3. A basis set of two functions is represented as a two-dimensional search space. Different combinations of the two functions result in regions of physiologically plausible hemodynamic responses and areas of nonphysiologically plausible areas. The addition derivative term of the HRF adjusts the time to peak to be early or late depending on whether it is positive or negative. Within the inset boxes are two examples of responses with a bimodal nature having no clear distinction of being "activated" or deactivated.

within bilateral calcarine and lingual gyri, bilateral hippocampus, right inferior, middle and superior frontal, right putamen, and left precentral. The later occurring responses (between 5 and $6 \mathrm{~s}$ ) were exclusively outside the visual cortex and mostly small clusters including bilateral inferior parietal, left hippocampus, left cerebellum, right inferior frontal, right cuneus, right temporal, right supramarginal, and right precuneus.

A group-level paired $t$-test was used to formally evaluate whether the use of the basis set was an improvement over using just the single double-gamma HRF. This analysis (results not shown) demonstrated that task-related signal change was significantly larger ( $p$ (FDRcorrected $)=0.05$, cluster extent $=20$ ) for the basis set model than for the single HRF model. Investigation of regions having significantly larger responses with the single HRF model demonstrated a single cluster in the right posterior horn of the lateral ventricle $(p($ uncorrected $)=0.001$, cluster extent $=20)$.

\section{Discussion}

The method described here is a simple and straightforward general framework for taking advantage of the added flexibility of basis sets at the group level while avoiding problematic implausible results. Our method incorporates the potential increased response magnitude from using any basis set and allows for a flexible means of limiting results to predefined physiologically plausible ones.

This study showed that estimated hemodynamic responses in the visual cortex to the visual stimuli tended to reach their peak amplitudes earlier than the canonical HRF's peak of $5 \mathrm{~s}$, as expected (Handwerker et al., 2004). When results were investigated for responses occurring earlier or later than $5 \mathrm{~s}$, all significant locations

Table 1

Physiological limits, corresponding contrast weights and the resultant angle.

\begin{tabular}{lllll}
\hline & Ratio & $\begin{array}{l}\text { Normalized } \\
\text { weights }\end{array}$ & $\begin{array}{l}\text { Contrast } \\
\text { weights }\end{array}$ & $\begin{array}{l}\text { Angle } \\
\text { (degrees) }\end{array}$ \\
\hline Later than $4 \mathrm{~s}$ & 0.44 & {$[0.920 .40]$} & {$[0.40-0.92]$} & 23.7 \\
Earlier than 5 s & 0 & {$[1.000 .00]$} & {$[0.001 .00]$} & 0 \\
Later than 5 s & 0 & {$[1.000 .00]$} & {$[0.00-1.00]$} & 0 \\
Earlier than 6 s & -0.34 & {$[0.95-0.32]$} & {$[0.320 .95]$} & -18.6 \\
\hline
\end{tabular}
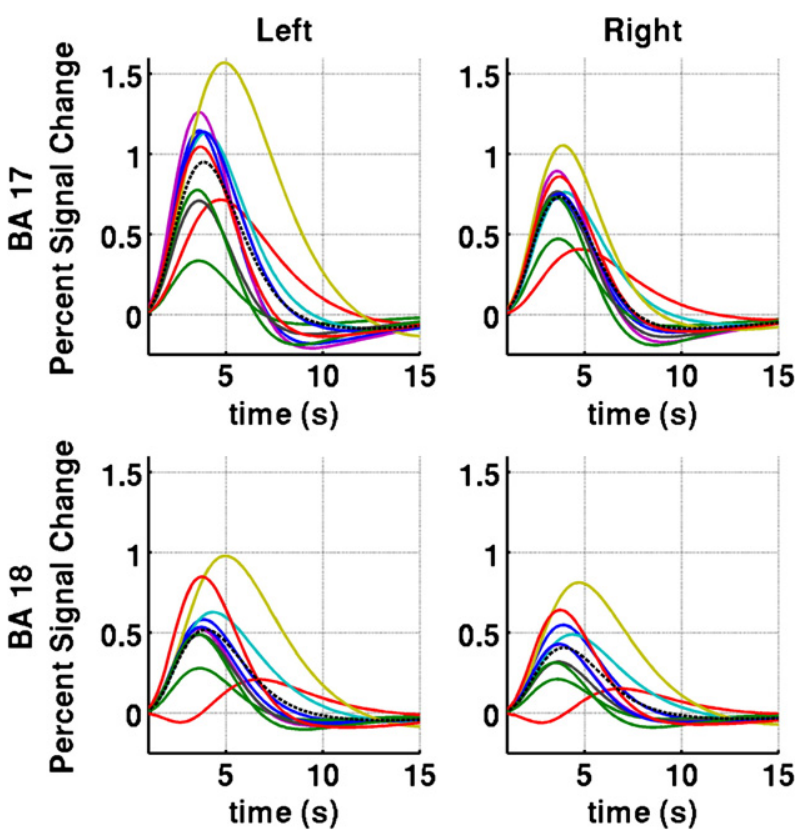

Fig. 4. Hemodynamic response estimates in the visual cortex. The estimated hemodynamic responses averaged over left and right Brodmann areas 17 and 18 of the visual cortex for each of the ten participants in this study (in color) and the group mean in a black dashed line. These results demonstrate that the majority of participants had responses which occurred earlier than the 5-s peak of the primary basis function.

within the visual cortex peaked within the range of 4 to $5 \mathrm{~s}$. The use of a basis set in this application allowed us to account for the latency differences from the canonical response and for inter- and intraindividual differences.

It is possible that performance of group-level parametric tests on the magnitude image may violate some assumptions of parametric statistics. Due to the small sample size in the current study, assessment of normality across participants in candidate voxels was not a reliable approach. Therefore, permutation tests were performed and results compared to parametric test results. Using the FMRIB Software Library (FSL) "randomise" program, a one-sample nonparametric test was performed on the magnitude images from each participant using 1024 permutations and the resultant probabilities were calculated voxelwise (Nichols and Holmes, 2002; Smith et al., 2004). For a one-sample $t$-test with $N=10$, only 1024 permutations are required for an exhaustive test due to redundancies in the shuffles. The resultant $t$-map from the parametric mapping approach was also converted to a $p$-map. The voxelwise comparison between the two approaches for $(1-p)$ values are shown in Fig. 6 . The scatter plot shows that the resultant probabilities from the two methods are comparable with a slight bias towards the nonparametric approach.

Table 2

Time to peak in seconds.

\begin{tabular}{llllll}
\hline \multirow{2}{*}{ Participant } & \multicolumn{2}{l}{ BA 17} & & \multicolumn{2}{l}{ BA 18} \\
\cline { 2 - 3 } & $\mathrm{L}$ & $\mathrm{R}$ & & $\mathrm{L}$ & $\mathrm{R}$ \\
\hline 1 & 3.60 & 3.65 & 3.85 & 3.85 \\
2 & 3.60 & 3.65 & 3.65 & 3.60 \\
3 & 4.70 & 4.75 & & 6.70 & 6.85 \\
4 & 3.95 & 3.95 & & 4.35 & 4.35 \\
5 & 3.60 & 3.55 & 3.65 & 3.70 \\
6 & 4.90 & 3.85 & & 4.95 & 4.70 \\
7 & 3.60 & 3.60 & 3.65 & 3.65 \\
8 & 3.85 & 3.70 & 3.70 & 3.65 \\
9 & 3.50 & 3.50 & 3.55 & 3.45 \\
10 & 3.70 & 3.70 & 3.75 & 3.75 \\
\hline
\end{tabular}




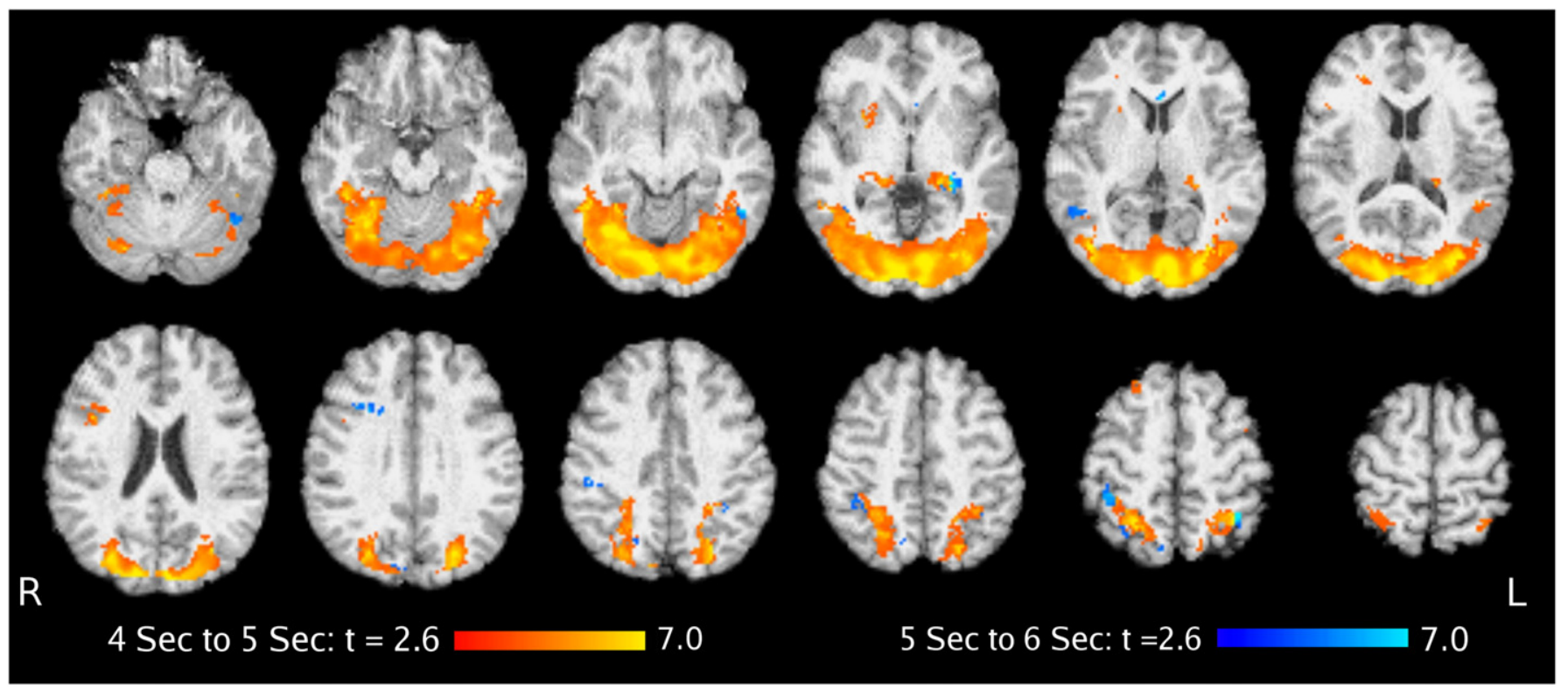

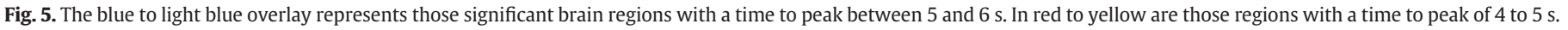

The current approach has the objective of capturing hemodynamic variation; however, the source of this variation may have a number of different origins (Henson et al., 2002) independent of inter- and intraparticipant time point variations (Aguirre et al., 1998). One dominant source of spatial variability is the temporal difference in BOLD responses in gray matter versus responses adjacent to larger vessels which can be thought of as a point spread response to neural activity (Lee et al., 1995; Pfeuffer et al., 2003; Saad et al., 2001). Another explanation is that variation in the BOLD response latencies may be neuronal in nature. Within the visual cortex, there is evidence that neuronal responses have different latencies across anatomical locations (Leopold et al., 2003; Ress and Heeger, 2003; Schmolesky et al., 1998). BOLD imaging may not be able to detect such variance in neural latencies (Ress and Heeger, 2003); it is possible that some

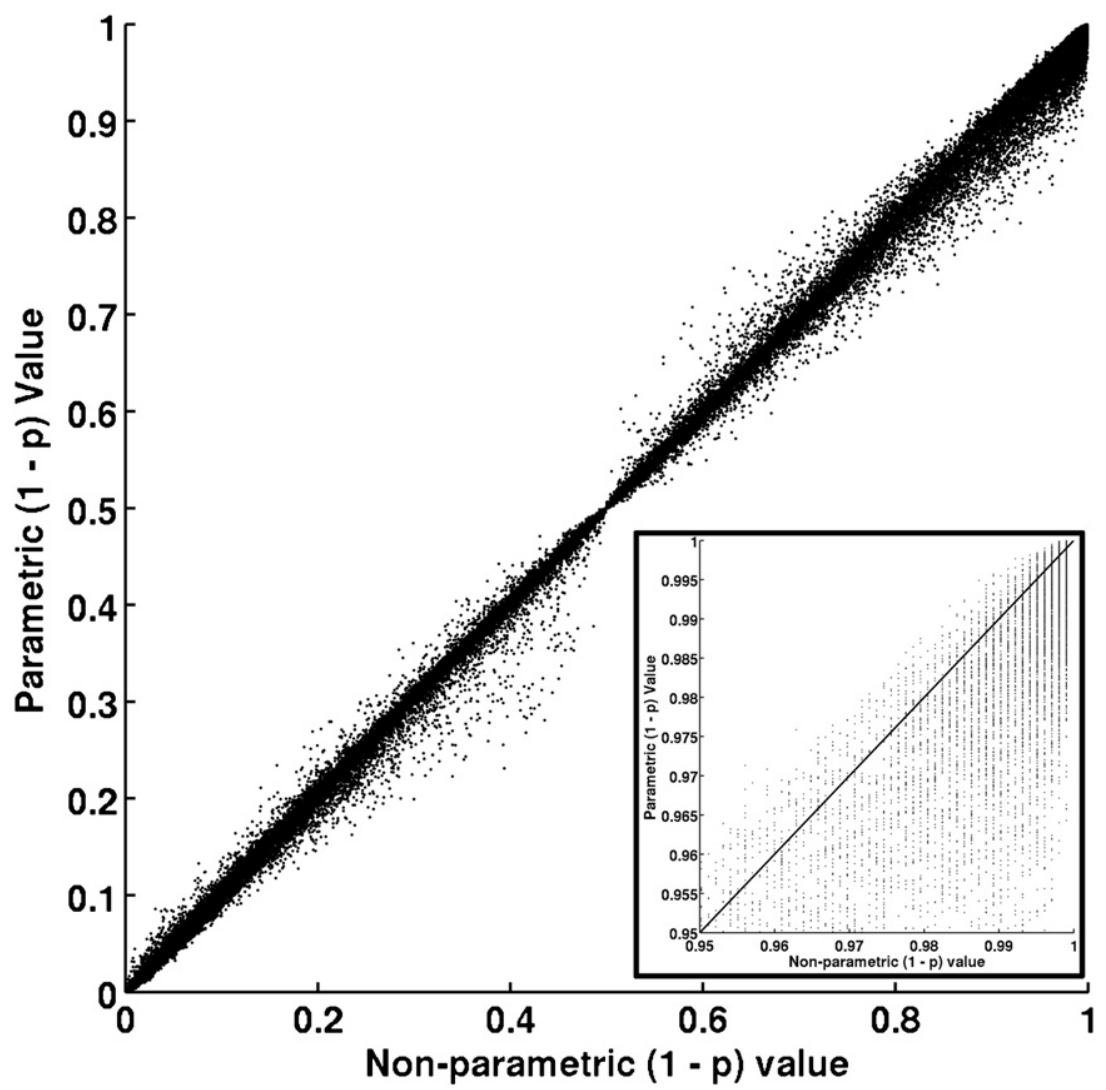

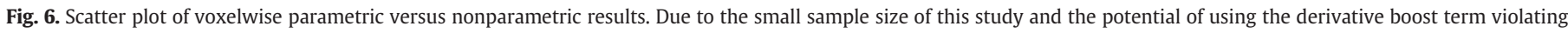

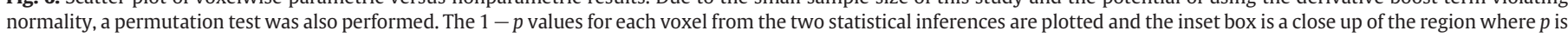
less than 0.05 . 
variation in the delay of a measured hemodynamic response may have a neuronal source. While the use of basis sets may capture this delay variance, they will not provide any evidence regarding its source. Therefore, detected latency variance in the estimated BOLD response must be interpreted with caution.

Although basis sets provide a means of detecting BOLD responses that vary within or across individuals, their use has two main limitations: (1) the means of performing statistical tests differs from standard $t$-tests on single regressors and (2) not all combinations of basis functions are of interest.

The simplest approach to account for both of these limitations is to allow the higher-order basis function(s) to capture variance in the data as 'regressor(s) of no interest,' thereby reducing the individual-level error term (Kiehl and Liddle, 2001). This approach may increase statistical values at the individual level but may not improve statistics when using group-level ordinary least squares. If analyses do not account for single subject-level variance at the group level, the extra variance accounted for at the single subject level does not translate to improved group-level results. If, however, single subject-level variance was brought forward to the group level, then the extra regressors may in fact improve group-level results (Beckmann et al., 2003).

Using the magnitude of the estimated regression weights of the basis functions allows for group-level analysis by combining information from the entire basis set. Therefore, analyses are performed on estimated HDRs that may vary across voxels and participants. The current approach is essentially a transformation into polar coordinates. The ratio of the weights is analogous to the calculation of the angle between the response projected onto the two basis functions (Friman et al., 2003; Worsley and Taylor, 2006). For comparison to these works, the angles for the different limits used here are presented in the last column of Table 1. The magnitude calculation is also analogous to an F-test, which similarly does not preserve the sign of the response (Calhoun et al., 2004; Worsley and Taylor, 2006). An obvious extension of our work is to a set of three basis functions and the use of spherical coordinates. The increase in complexity will lie in the number of contrasts required to define limitations of the model fits. Two basis functions require two contrasts to define a range of interest, whereas three basis functions required a minimum of four contrasts to designate a range of interest.

Although the data used to demonstrate our procedure only investigated positive BOLD responses, the physiological limits may be altered to investigate negative BOLD responses (deactiviations) by flipping the sign of the first number in each contrast weight in Table 1. The ranges of interest may be defined for any range around the circle shown in Fig. 3 as long as the limitations on sensitivity demonstrated in Fig. 1B are considered. With increased interest in negative BOLD responses, relative to baseline, the question arises as to whether the positive and negative BOLD responses are identical. Work in the epilepsy literature demonstrates the importance of such questions (Salek-Haddadi et al., 2006).

The limits used here were derived from the linear range of the mapping of the relationship between basis function weights and the resultant physiologic parameter of interest similar to that used in Henson et al. (2002). However, this approach is not limited to testing for time to peak latencies using this basis set. Similar mappings may be made in relation to other physiologic parameters such as the size of the undershoot, width of the response or parameters derived from other basis sets. One alternative basis set derived by Liao et al. (2002) expands the detectable latency range beyond that of the basis set used here. The result is an expanded region of sensitivity for which latencies can be measured. The importance of the work by Liao et al. (2002) is that they designed a basis set with increased sensitivity to a specific physiological aspect of the HDR, its delay.

As an example of our approach, a basis set can be designed to specifically investigate, or account for, the variability of the poststimulus response found in aging (D'Esposito et al., 2003; Wierenga et al.,
2008). To address these questions a new basis set is created which is specifically sensitive to the poststimulus undershoot variability. This is easily implemented using the "Make FLOBS" tool provided with the FSL package (Woolrich et al., 2004). This toolbox creates a large set $(N=10,000)$ of plausible hemodynamic responses that vary within specified ranges of values for each temporal phase of the response. To exemplify the process the following parameters (as defined in the "Make FLOBS" tool) were used: time to initial rise, $m_{1}=0.5 \mathrm{~s}$; rise time, $m_{2}=4.5 \mathrm{~s}$; fall time, $m_{3}=10.5 \mathrm{~s}$; return to baseline range, $m_{4}=$ [3-8] s; proportion of poststimulus size to response range, $c=$ [0-0.5]. Using these values, the first two principal components spanning the set are used as the basis set, see Fig. 7A. Ratios of these two orthogonal functions are then mapped onto the resultant proportion of poststimulus undershoot to response size, Fig. 7B. A response with no undershoot would be fit with a ratio of estimated beta values of -0.27 and a poststimulus undershoot one half the size of the response would have a ratio of estimated beta values of 0.26 . Therefore, a range of interest could be between $r_{1}=-0.27$ and $r_{2}=0.26$ (responses with ratios to the right of -0.27 and to the left of 0.26 on Fig. 7B). After normalizing and rotating these values, the two contrasts are $c_{1}=\left[\begin{array}{ll}0.26 & 0.96\end{array}\right]$ and $c_{2}=\left[\begin{array}{l}-0.250 .97\end{array}\right]$.

This approach of designing a basis set to address a specific physiological or clinical question may improve the sensitivity of fMRI for further use in more clinical applications (Lemieux et al., 2008; Matthews et al., 2006; Salek-Haddadi et al., 2003). As an example, studies of epileptiform discharges with BOLD fMRI demonstrated large variability in the shape of the HDR proximally and distally from discharge sites (Lemieux et al., 2008; Salek-Haddadi et al., 2006). In the study by Salek-Haddadi et al. (2006), the variability was assessed using a set of Fourier basis functions. Inspection of each individual's estimated HDR from the epileptic discharge site demonstrated large variability in the time to peak delays, the undershoot amplitude, the undershoot time, and the ratio of undershoot to peak amplitudes. Some of the estimated delay values are outside the linear range of the canonical model of the HRF and its first derivative basis set (Henson et al., 2002), thus making the Fourier basis set more appropriate.

Fourier and finite impulse response (FIR) basis offer great flexibility by assuming minimal a priori information for the expected HDR shape and require alternate approaches to increase interpretability. One method of implementing adjustable physiological limitations is via temporal regularization. This incorporates physiological knowledge of the signal into the estimation of the HRF, i.e., the HRF starting and ending at zero and being smooth. Some approaches include the use of smooth FIR filters (Goutte et al., 2000), using a Bayesian framework with temporal priors (Marrelec et al., 2003) or a deterministic approach using the regularization by Tikhonov and Arsenin (1977) (see Casanova et al., 2008 for a review). These approaches may have their benefits over the presented approach; however, their level of sophistication is much greater.

Another approach to interpret results from using an FIR basis set is with area under the curve (AUC) measures where the sum of the basis function fits is summed within a predefined window.. This measure may be confounded for between group comparisons if the HDR differs as a function of group. One example using FIR basis sets in the aging literature found that an elder group of adults had a limited poststimulus undershoot, as compared to their younger counterparts who had large poststimulus negative deflections (Wierenga et al., 2008). The use of the AUC measure would potentially be biased towards the elder group who showed smaller poststimulus negative deflections and thus less negative values than the young group. The result from this study showed larger AUC values in elder adults than in young adults and was interpreted as larger BOLD responses for the elderly. Another example of potentially difficult to interpret results from a study using an FIR basis set used the AUC measure for a 4-s window (Kable and Glimcher, 2007). While this approach may be appropriate with healthy young populations, it may introduce bias for 
A

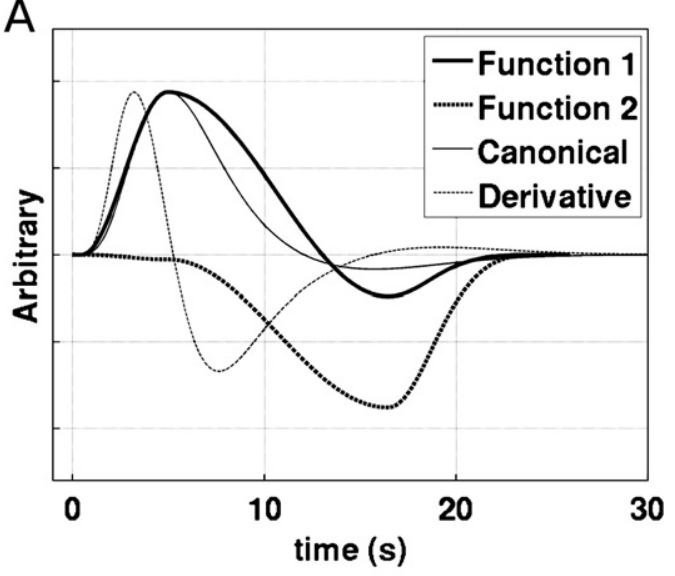

B

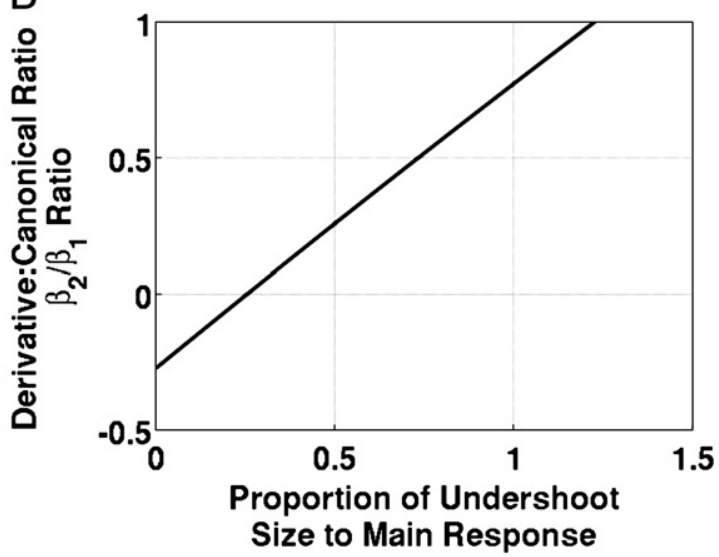

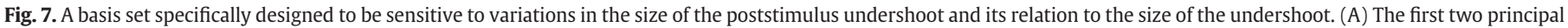

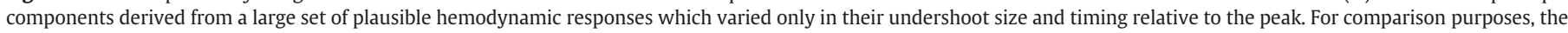

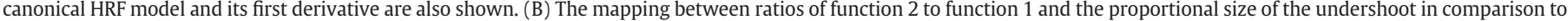
the main response.

between-group comparisons which systematically differ in the timing or shape of their hemodynamic responses.

An alternative to using basis sets to capture individual variance in the HDR is the use of participant-specific estimated HRFs (D'Esposito et al., 1999; Zarahn et al., 1997). One drawback is that this approach is no more sensitive to spatial variations in the HDR then the use of a canonical HRF. There is, however, the possibility of developing a participant-specific basis set model of an individual's HDR. One approach would be to estimate a participant-specific HRF and then vary its temporal delay systematically as in the approach by Liao et al. (2002). A subsequent PCA would then create the participant-specific basis set. A second approach is to perform trial averages, or some other means of estimating the HDR, across all voxels in a region of interest and then perform a PCA on the result. The use of participantspecific hemodynamic basis sets may be an approach which takes advantage of the multiple advances in this area and thus may improve sensitivity in the face of spatial and participant variability resulting from normal, developmental, degenerative, or pathological variability.

\section{Acknowledgments}

The authors are grateful to Martin Lindquist for helpful discussion and expertise. This research was supported by the National Institute of Aging, grant number 1K01AG21548 to M.H. Tabert.

\section{Appendix 1}

The analyses used the model:

$y_{t}=\hat{\beta}_{1} x_{t}+\hat{\beta}_{2} \frac{\partial x_{t}}{\partial t}+\hat{\beta}_{3}+\varepsilon_{t}$

where the regressor

$x_{t}=s_{t} * h_{t}$

and $s_{t}$ represents the stimulus "box-car" model, $h_{t}$ represents the double-gamma model of the hemodynamic impulse response function, and $*$ refers to the convolution/filtering operation. The equation for the double-gamma model (Handwerker et al., 2004; Lindquist and Wager, 2007, Glover, 1999; Liao et al., 2002) as described in Lindquist et al. (2009) is:

$h(t)=\left(\frac{t^{\alpha_{1}-1} \beta_{1}^{\alpha_{1}} e^{-\beta_{1} t}}{\Gamma\left(\alpha_{1}\right)}-c \frac{t^{\alpha_{2}-1} \beta_{2}^{\alpha_{2}} e^{-\beta_{2} t}}{\Gamma\left(\alpha_{2}\right)}\right)$ where $t$ is time, $\alpha_{1}=6, \alpha_{2}=16, \beta_{1}=\beta_{2}=1, c=1 / 6$, and $\Gamma$ is the gamma function.

Assuming that the regressors are normalized such that:

$\sum_{t}^{N}\left(\frac{\partial x_{t}}{\partial t}\right)^{2}=1 \quad \sum_{t}^{N} x_{t}^{2}=1 \quad \sum_{t}^{N} x_{t} \frac{\partial x_{t}}{\partial t}=0$

the "derivative boost" is calculated as:

$H=\sqrt{\left(\hat{\beta}_{1}^{2}+\hat{\beta}_{2}^{2}\right)}$

However, if the regressors are not normalized as specified above, the estimated betas, or contrasts, may be normalized by the sum of squares of the regressors after model estimation.

Therefore, letting:

$y_{t}=\tilde{\beta}_{1} \tilde{x}_{t}+\tilde{\beta}_{2} \frac{\partial \tilde{x}_{t}}{\partial t}+\tilde{\beta}_{3}+\varepsilon_{t}$

where the tilde $(\sim)$ represents that these values are unnormalized. The normalized values of beta are calculated as:

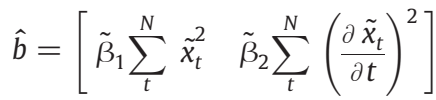

Likewise, the contrast:

$c=\left[\begin{array}{ll}c_{1} & c_{2}\end{array}\right]$

where:

$\hat{b}=\left[\begin{array}{ll}\hat{\beta}_{1} & \hat{\beta}_{2}\end{array}\right] \quad \tilde{b}=\left[\tilde{\beta}_{1} \tilde{\beta}_{2}\right]$

is similarly scaled such that the resultant contrast-weighted betas are normalized:

$c \hat{b}=c\left[\begin{array}{cc}\sum_{t}^{N} \tilde{x}_{t}^{2} & 0 \\ 0 & \sum_{t}^{N}\left(\frac{\partial \tilde{x}_{t}}{\partial t}\right)^{2}\end{array}\right] \tilde{b}$ 
Therefore, the derivative boost calculated using an un-normalized design matrix as:

$H=\sqrt{\left(\hat{\beta}_{1}^{2} \sum_{t}^{N} \tilde{x}_{1}^{2}+\hat{\beta}_{2}{ }^{2} \sum_{t}^{N}\left(\frac{\partial \tilde{x}_{t}}{\partial t}\right)^{2}\right)}$

Of note is that the normalization described here differs slightly from the "derivative boost" calculation previously described (Calhoun et al., 2004). The difference is that the sign of the primary basis function weight is not used. The sign of the response is determined from contrast weight restrictions. The information regarding the sign is therefore not lost but is incorporated in a different step of the analysis. The code for performing the magnitude image calculation (A1.2) or if needed with adjustments for a non-normalized design matrix (A1.4) for use with SPM or FSL is available at the Web site: http://cumc.columbia.edu/dept/sergievsky/cnd/steffener.html.

\section{Appendix 2}

Calculations of the contrasts to test are derived from the ratio of beta estimates of interest as described below. Once a limit of interest is chosen, for example, from Fig. 1B, its corresponding ratio is found. Then, the range of interest is decided: either to the left or right of this limit. The two limits used in the current work are used as examples below. A tilde $(\sim)$ above a contrast vector means that this is not the final value. The final contrast vectors are designated with a bar.

For the later than $4 \mathrm{~s}$ limit, the ratio is

$r_{1}=0.44$

Create a contrast vector from the ratio:

$\tilde{c}_{1}=\left[\begin{array}{ll}1 & 0.44\end{array}\right]$

Normalize the contrast:

$\tilde{c}_{1}=\frac{\tilde{c}_{1}}{\sqrt{\left(c_{1}^{2}(1)+c_{1}^{2}(2)\right)}}$

$\tilde{c}_{1}=\left[\begin{array}{ll}0.92 & 0.40\end{array}\right]$

The region of interest in relation to this ratio is to its right; therefore, the contrast is rotated as:

$\tilde{c}_{1}=\left[\begin{array}{ll}0.92 & 0.40\end{array}\right]\left[\begin{array}{cc}0 & -1 \\ 1 & 0\end{array}\right]$

Final contrast:

$\bar{c}_{1}=\left[\begin{array}{ll}0.40 & -0.92\end{array}\right]$

For the earlier than $6 \mathrm{~s}$ limit, the ratio is

$r_{2}=-0.34$

Create a contrast vector from the ratio:

$\tilde{c}_{2}=\left[\begin{array}{ll}1 & -0.34\end{array}\right]$

Normalize the contrast:

$\tilde{c}_{2}=\frac{\tilde{c}_{2}}{\sqrt{\left(c_{2}^{2}(1)+c_{2}^{2}(2)\right)}}$

$\tilde{c}_{2}=\left[\begin{array}{ll}0.95 & -0.32\end{array}\right]$
The region of interest in relation to this ratio is to its right; therefore, the contrast is rotated as:

$\tilde{c}_{2}=\left[\begin{array}{ll}0.95 & -0.32\end{array}\right]\left[\begin{array}{cc}0 & 1 \\ -1 & 0\end{array}\right]$

Final contrast:

$\bar{c}_{2}=\left[\begin{array}{ll}0.32 & 0.95\end{array}\right]$

Of important note is that the contrasts calculated here use a normalized design matrix (meets the constraints in (A1.1)). If the design matrix used does not meet these criteria then the above contrasts ((A2.1) and (A2.2)) need to be adjusted as in (A1.3). The code for calculating the contrast weights for a given ratio while also performing adjustments when a non-normalized design matrix is used is available at the Web site: http://cumc.columbia.edu/dept/ sergievsky/cnd/steffener.html.

\section{References}

Aguirre, G.K., Zarahn, E., D'Esposito, M., 1998. The variability of human, BOLD hemodynamic responses. NeuroImage 8, 360-369.

Ances, B.M., Liang C.L. Leontiev, O., Perthen, J.E., Fleisher, A.S., Lansing A.E., Buxton, R. B. 2009. Effects of aging on cerebral blood flow, oxygen metabolism, and blood oxygenation level dependent responses to visual stimulation. Hum. Brain. Mapp. 30, 1120-1132.

Beckmann, C.F., Jenkinson, M., Smith, S.M., 2003. General multilevel linear modeling for group analysis in FMRI. NeuroImage 20, 1052-1063.

Calhoun, V.D., Stevens, M.C., Pearlson, G.D., Kiehl, K.A., 2004. fMRI analysis with the general linear model: removal of latency-induced amplitude bias by incorporation of hemodynamic derivative terms. Neurolmage 22, 252-257.

Casanova Ryali, S., Serences, J., Yang, L., Kraft, R., Laurienti, P.J., Maldjian, J.A., 2008. The impact of temporal regularization on estimates of the BOLD hemodynamic response function: a comparative analysis. Neurolmage 40, 1606-1618.

D'Esposito, M., Postle, B.R., Ballard, D., Lease, J., 1999. Maintenance versus manipulation of information held in working memory: an event-related fMRI study. Brain. and. Cognition. 41, 66-86.

D'Esposito, M., Deouell, L.Y., Gazzaley, A., 2003. Alterations in the BOLD fMRI signal with ageing and disease: a challenge for neuroimaging. Nat. Rev. Neurosci. 4, 863-872.

Ford, J.M., Johnson, M.B., Whitfield, S.L., Faustman, W.O., Mathalon, D.H., 2005. Delayed hemodynamic responses in schizophrenia. NeuroImage 26, 922-931.

Friman, O., Borga, M., Lundberg, P., Knutsson, H., 2003. Adaptive analysis of fMRI data. Neurolmage 19, 837-845.

Friston, K.J., Fletcher, P., Josephs, O., Holmes, A., Rugg, M.D., Turner, R., 1998. Eventrelated fMRI: characterizing differential responses. NeuroImage 7, 30-40.

Glover, G.H., 1999. Deconvolution of impulse response in event-related BOLD fMRI. Neurolmage 9, 416-429.

Goutte, C., Nielsen, F.A., Hansen, L.K., 2000. Modeling the haemodynamic response in fMRI using smooth FIR filters. IEEE. Trans. Med. Imaging. 19, 1188-1201.

Handwerker, D.A., Ollinger, J.M., D'Esposito, M., 2004. Variation of BOLD hemodynamic responses across subjects and brain regions and their effects on statistical analyses. NeuroImage 21, 1639-1651.

Henson, R.N., Price, C.J., Rugg, M.D., Turner, R., Friston, K.J., 2002. Detecting latency differences in event-related BOLD responses: application to words versus nonwords and initial versus repeated face presentations. NeuroImage 15, 83-97.

Kable, J.W., Glimcher, P.W., 2007. The neural correlates of subjective value during intertemporal choice. Nat. Neurosci. 10, 1625-1633.

Kiehl, K.A., Liddle, P.F., 2001. An event-related functional magnetic resonance imaging study of an auditory oddball task in schizophrenia. Schizophr. Res. 48, 159-171.

Lee, A.T., Glover, G., Meyer, C.H., 1995. Discrimination of large venous vessels in timecourse spiral blood-oxygen-level-dependent magnetic-resonance functional neuroimaging. Magn. Reson. Med. 33, 745-754.

Lemieux, L., Laufs, H., Carmichael, D., Paul, J.S., Walker, M.C., Duncan, J.S., 2008. Noncanonical spike-related BOLD responses in focal epilepsy. Hum. Brain Mapp. 29, 329-345.

Leopold, D.A., Murayama, Y., Logothetis, N.K., 2003. Very slow activity fluctuations in monkey visual cortex: implications for functional brain imaging. Cereb. Cortex 13, 422-433.

Liao, C.H., Worsley, K.J., Poline, J.B., Aston, J.A.D., Duncan, G.H., Evans, A.C., 2002. Estimating the delay of the fMRI response. Neurolmage 16, 593-606.

Lindquist, M.A., Wager, T.D., 2007. Validity and power in hemodynamic response modeling: a comparison study and a new approach. Hum. Brain Mapp. 28, 764-784

Lindquist, M.A., Meng Loh, J., Atlas, L.Y., Wager, T.D., 2009. Modeling the hemodynamic response function in fMRI: efficiency, bias and mis-modeling. Neurolmage 45, S187-S198.

Marrelec, G., Benali, H., Ciuciu, P., Pelegrini-Issac, M., Poline, J.B., 2003. Robust Bayesian estimation of the hemodynamic response function in event-related BOLD fMRI using basic physiological information. Hum. Brain Mapp. 19, 1-17. 
Matthews, P.M., Honey, G.D., Bullmore, E.T., 2006. Applications of fMRI in translational medicine and clinical practice. Nat. Rev. Neurosci. 7, 732-744.

Nichols, T.E., Holmes, A.P., 2002. Nonparametric permutation tests for functional neuroimaging: a primer with examples. Hum. Brain Mapp. 15, 1-25.

Pfeuffer, J., McCullough, J.C., Van de Moortele, P.-F., Ugurbil, K., Hu, X., 2003. Spatial dependence of the nonlinear BOLD response at short stimulus duration. NeuroImage 18, 990-1000.

Ress, D., Heeger, D.J., 2003. Neuronal correlates of perception in early visual cortex. Nat. Neurosci. 6, 414-420.

Rorden, C., Brett, M., 2000. Stereotaxic display of brain lesions. Behav. Neurol. 12, 191-200.

Saad, Z.S., Ropella, K.M., Cox, R.W., DeYoe, E.A., 2001. Analysis and use of FMRI response delays. Hum. Brain Mapp. 13, 74-93.

Salek-Haddadi, A., Friston, K.J., Lemieux, L., Fish, D.R., 2003. Studying spontaneous EEG activity with fMRI. Brain Res. Brain Res. Rev. 43, 110-133.

Salek-Haddadi, A., Diehl, B., Hamandi, K., Merschhemke, M., Liston, A., Friston, K., Duncan, J.S., Fish, D.R., Lemieux, L., 2006. Hemodynamic correlates of epileptiform discharges: an EEG-fMRI study of 63 patients with focal epilepsy. Brain Res. 1088, 148-166.

Schmolesky, M.T., Wang, Y., Hanes, D.P., Thompson, K.G., Leutgeb, S., Schall, J.D., Leventhal, A.G., 1998. Signal timing across the macaque visual system. J. Neurophysiol. 79, 3272-3278.

Smith, S.M., Jenkinson, M., Woolrich, M.W., Beckmann, C.F., Behrens, T.E.J., JohansenBerg, H., Bannister, P.R., De Luca, M., Drobnjak, I., Flitney, D.E., Niazy, R.K., Saunders, J., Vickers, J., Zhang, Y., De Stefano, N., Brady, J.M., Matthews, P.M., 2004. Advances in functional and structural MR image analysis and implementation as FSL NeuroImage 23, S208-S219.

Stevens, M.C., Calhoun, V.D., Kiehl, K.A., 2005. Hemispheric differences in hemodynamics elicited by auditory oddball stimuli. Neurolmage 26, 782-792.

Tikhonov, A.N., Arsenin, V.Y., 1977. Solution of Ill-posed Problems. W.H. Winston, Washington D.C.

Tzourio-Mazoyer, N., Landeau, B., Papathanassiou, D., Crivello, F., Etard, O., Delcroix, N., Mazoyer, B., Joliot, M., 2002. Automated anatomical labeling of activations in SPM using a macroscopic anatomical parcellation of the MNI MRI single-subject brain. Neurolmage 15, 273-289.

Wierenga, C.E.., Benjamin, M., Gopinath, K.,Perlstein, W.M., Leonard, C.M., Rothi, L.J., Conway, T., Cato, M.A., Briggs, R., Crosson, B., 2008. Age-related changes in word retrieval: role of bilateral frontal and subcortical networks. Neurobiol. Aging. 29, 436-451.

Woolrich, M.W., Behrens, T.E., Smith, S.M., 2004. Constrained linear basis sets for HRF modelling using variational Bayes. NeuroImage 21, 1748-1761.

Woolrich, M.W., Chiarelli, P., Gallichan, D., Perthen, J., Liu, T.T., 2006. Bayesian inference of hemodynamic changes in functional arterial spin labeling data. Magn. Reson. Med. 56, 891-906.

Worsley, K.J., Taylor, J.E., 2006. Detecting fMRI activation allowing for unknown latency of the hemodynamic response. NeuroImage 29, 649-654.

Yang, Y., Engelien, W., Pan, H., Xu, S., Silbersweig, D.A., Stern, E., 2000. A CBF-based event-related brain activation paradigm: characterization of impulse-response function and comparison to BOLD. Neurolmage 12, 287-297.

Zarahn, E., Aguirre, G.K., D'Esposito, M., 1997. Empirical analyses of BOLD fMRI statistics. NeuroImage 5, 179-197. 